\title{
Granular Cell Ameloblastoma: Retrospective Clinical and Histopathological Findings of Case Series and Review of Literature
}

\author{
Hoyos AM*, Teshima THN, Dias CD, Pinto CAL, \\ Coutinho-Camillo CM and Lourenço SV \\ Department of Stomatology, University of Sao Paulo, \\ Brazil \\ *Corresponding author: Ana Maria HoyosCadavid, \\ Department of Stomatology, School of Dentistry, Av. Prof \\ Lineu Prestes 2227, University of Sao Paulo, Sao Paulo, \\ Brazil
}

Received: September 05, 2018; Accepted: October 09, 2018; Published: October 16, 2018

\begin{abstract}
Granular Cell Ameloblastoma (GCA) is classified as an unusual histological subtype of solid/multicystic ameloblastoma, characterized by granular changes of stellate-like cells located within the inner portion of epithelial follicles. Studies have revealed that cytoplasmatic granularity is caused by lysosomal overload; however, the mechanism involved remains poorly understood. Here we report eight cases of granular cell ameloblastoma, in the posterior region of the mandible. The age of the patients included in this case series ranges between $35-64$ years old, and $87.5 \%$ occurred in non-Caucasians, with a slight gender predilection for men (62.5\%). There was no evidence of recurrence and the majority of the cases were treated with surgical resection (87.5\%). All tumors displayed histopathological features consistent with the diagnosis of granular cell tumor
\end{abstract}

Keywords: Ameloblastoma; Odontogenic Tumors; Pathology

\section{Clinical Relevance}

This paper presents the clinical and histopathological findings of eight cases of granular cell ameloblastoma, which is a rare tumor that represents only $3-5 \%$ of all ameloblastomas and predisposes to important risk of recurrence.

\section{Introduction}

Odontogenic tumors comprise a complex group of lesions of diverse histopathological subtypes and clinical behavior, which can originate from tissue remnants of tooth formation. Ameloblastoma is a true neoplasm that particularly rises from the enamel organ type tissue with a slow growth pattern, although with infiltrative and recurrent potential, and it corresponds to the second most common odontogenic tumor (11\%) [1,2]. This lesion is usually found almost exclusively in the lower jaw, near the angle of the mandible, and occur more often between the third and fifth decades of life $[1,3]$.

According to the clinical and radiographic features, ameloblastomas are classified into conventional solid or multicystic, extra osseous or peripheral, desmoplastic and unicystic types [4].They can display distinctive histological findings mainly characterized by the presence of cystic areas composed of peripheral columnar cells in a palisaded basal cell layer that surrounds hyper chromatic granular cells. There are six histological variants known as follicular, plexiform (most common subtypes), acanthomatous, desmoplastic, granular and basal cell $[3,4]$.

Granular cell ameloblastoma (GCA) is a rare type of ameloblastoma that represents only 3-5\% of all histological subtypes. GCA is mostly found as a combination of different histological patterns, particularly the follicular variant $[1,5]$.This tumor was first observed and described by Krompecher in 1918 when it was named as pseudo xanthomatous cells $[1,2]$. It appears to be aggressive in nature, with a marked propensity for recurrence and can progress to metastasis $[2,6]$.

Histologically, GCA is characterized by the presence of granular cells, which are usually small structures of around $1 \mu \mathrm{m}$ compared to rare giant granular cells that are up to $30 \mu \mathrm{m}$. These cells appear typically within the central area of ameloblastic tumor follicles, progressively replacing the stellate reticulum, which may also extend to the peripheral columnar or cuboidal cells [2]. The granular cell shape can vary from the most common round to cuboidal, columnar or angular, in which the nuclei are pyknotic and hyper chromatic and the cytoplasm is filled with acidophilic granules, identified as lysosomal aggregates [2,7]. The presence of granular cells represents either a transitional or a matured stage in the life cycle of ameloblastomas. They start as normal stellate reticulum like cells, lead to the production of cytoplasmic granules and finally follow towards tissue degeneration and formation of cystic areas [5].

The current treatment for GCA is similar to other subtypes of ameloblastomas, in which radical surgical methods and accurate preoperative diagnosis. Regular follow-up management is recommended as conservative treatments as enucleation or curettage exhibit high recurrence rate [2]. Previous studies suggest that GCA represents more aggressive lesions, whereas others propose that GCA is just a transitional phase during the pathological course of ameloblastomas, therefore not requiring special attention $[1,2]$.

The prognosis of GCA is considered favorable when early treatment is performed; however recurrence rate of over $30 \%$ has already been reported, implying potential unknown aggressive behavior of GCA [8]. A proper regular follow-up is therefore recommended, as recurrence has been shown to happen up to eight years after the initial treatment $[1,2]$. The uncertain clinical behavior reported for this type of ameloblastoma emphasizes the importance 


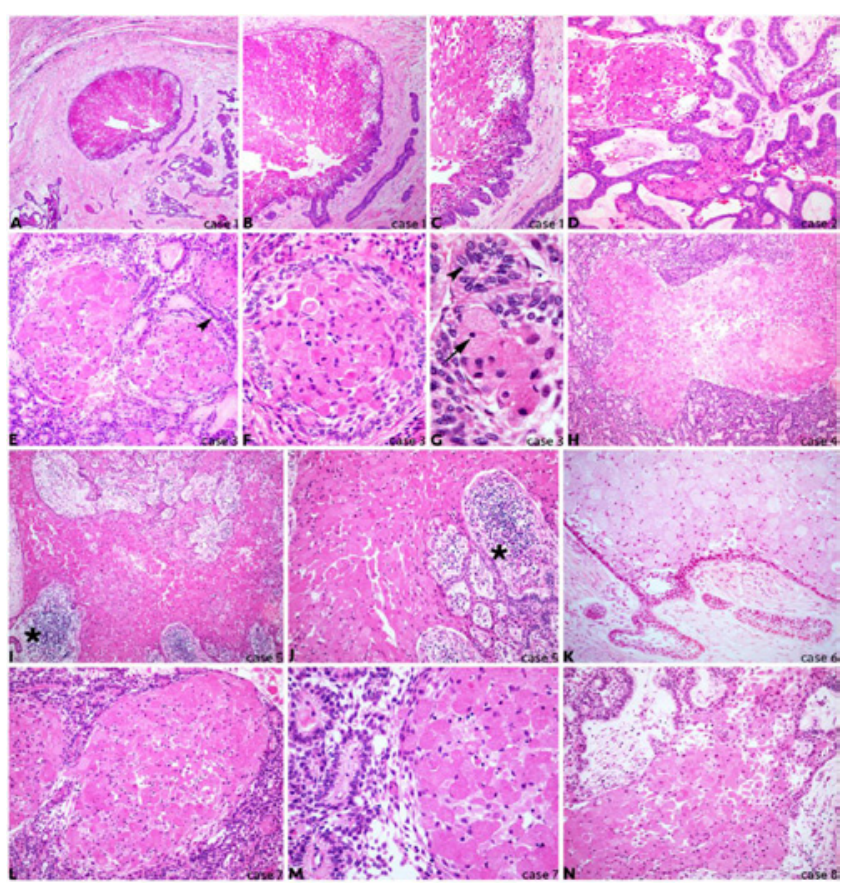

Figure 1: Histological sections of retrospective granular cell ameloblastoma case series illustrate the main features of this rare subtype of odontogenic tumor Figure 1A-1C: Case 1 with localized granular cell cluster surrounded by the typical palisaded epithelium (original magnification A 40X, B 100X, C 200X); Figure 1D: Case 2 shows widespread typical ameloblastic epithelium interlaced within areas of granular cells (200X);

Figure 1E-1G: Case 3 highlights classical granular cell content with varied cell shape, pyknotic nuclei (arrow) and palisaded epithelial basal cell layer surrounding granular cell clusters (arrowheads) (E 200X, F 400X, G 630X);

Figure 1H: Case 4 shows extensive granular cell cluster within ameloblastic epithelium (100X);

Figure 1I,1J: Case 5 presents wide granular cell content in between epithelial and inflammatory tissue (asterisks) (I 100X, J 200X);

Figure 1K: Case 6 illustrates the epithelial boundary of a granular cell cluster within a mature connective tissue (200X);

Figure 1L,1M: Case 7 shows granular cell nests surrounded by highly dense and proliferative ameloblastic epithelium (L 200X, M 400X);

Figure 1N: Case 8 displays extensive granular cell cluster with partially absent epithelial periphery (200X).

of better understanding its pathogenesis and clinical impact, and this article therefore aims to report the clinical and histological features of a retrospective case series of eight mandibular GCA.

\section{Case Series}

Given that GCA represents a rare pathological entity and that there are only few reports in the literature comparing their clinical and histopathological characteristics, a retrospective study of eight patients was performed. Clinical, demographic and histopathological data of all GCA diagnosed in the Department of Pathological Anatomy of the Hospital AC Camargo Cancer Center, SP Brazil, from 1953 to 2001, were evaluated according to the approval of the Ethics Committee In Research of the Faculty of Dentistry of the University of São Paulo - USP (protocol number 171/08;FR-216880).

The clinical features of all patients were obtained from their medical records, which include age, ethnicity, gender, and location of the lesion, treatment, and recurrence, radiographic and histopathological findings, shown in (Table 1). This retrospective analysis of GCA case series included eight patients ranging from 35 to 64 years old (average age of 46.8 years) with a slight predilection for men (62.5\%) and for non-Caucasians (87.5\%). The majority of the cases were treated with surgical resection $(87.5 \%)$ that presented no evidence of recurrence, provided with a follow-up of more than 3 years. All GCA included in this study was found at the posterior region of the mandible particularly near the angle, with a multilocular radiographic pattern.

Paraffin-embedded sections of all eight cases were stained with haematoxylin and eosin to further morphological analysis as shown in (Figure 1A-N). All cases presented a tumor area composed of granular cells with small and hyper chromatic nuclei (high magnification on (Figure 1F,1G) arrow) surrounded by stratified ameloblastic epithelium arranged in palisaded basal cell layer ((Figure 1E,1G), arrowheads). The granular cells were usually packed and distributed in one or multiple clusters throughout the epithelium, forming islands of tumor mass, sometimes also presenting great amount of inflammatory cells ((Figure 1I,1J), asterisks). There was a variation of both size and shape of these granular cells within the clusters, mostly round and presenting large cytoplasm. The connective tissue wrapping the tumor area was well-developed and prominently poorly vascularised. Cell pleomorphism and mitosis were absent.

These eight case-series of GCA showed the presence of multiple typical granular cells in the centre of the neoplastic nests, where the peripheral palisaded columnar or cuboidal cells were totally or partially limiting the tumor areas. The differential diagnosis included granular cell myoblastoma and granular cell ameloblastic fibroma, however the histopathological findings associated with the clinical and multilocular radiographic features resulted in the final diagnosis of GCA for all cases. 
Table 1: Retrospective clinical data of eight patients from AC Camargo Cancer Centre diagnosed with Granular Cell Ameloblastoma.

\begin{tabular}{|c|c|c|c|c|c|c|c|}
\hline Case & Age & Ethnicity & Gender & Location & Treatment & Recurrence & X-Ray findings \\
\hline $\mathbf{1}$ & 35 & Non-Caucasian & F & Mandible & Curettage & Notdetected \\
\hline $\mathbf{2}$ & 36 & Non-Caucasian & F & Mandible & Surgicalresection & Notdetected & Multilocular \\
\hline $\mathbf{3}$ & 48 & Non-Caucasian & M & Mandible & Surgicalresection & Notdetected & Multilocular \\
\hline $\mathbf{4}$ & 60 & Non-Caucasian & M & Mandible & Surgicalresection & Notdetected & Multilocular \\
\hline $\mathbf{5}$ & 57 & Non-Caucasian & M & Mandible & Surgicalresection & Multilocular \\
\hline $\mathbf{6}$ & $\mathbf{2 7}$ & Non-Caucasian & F & Mandible & Surgicalresection & Notdetected & Multilocular \\
\hline $\mathbf{7}$ & 64 & Caucasian & M & Mandible & Surgicalresection & Notdetected & Multilocular \\
\hline $\mathbf{8}$ & 48 & Non-Caucasian & M & Mandible & Surgicalresection & Notdetected & Multilocular \\
\hline
\end{tabular}

\section{Discussion}

Granular cell ameloblastoma is a rare and unusual histological variant of the odontogenic tumor ameloblastoma, representing less than $5 \%$. Reichart et al, have reported in an extensive review study from 1960 to 1993 only 56 cases of GCA out of a total of 1593 cases of ameloblastomas (3.5\%) [8]. Likewise Kameyama et al. in a clinic ophatological study observed only one GCA out of 77 ameloblastomas included in the study [9].

A case series published by Hartmann et al. evaluated all radiograph and clinical aspects of 20 patients from the United States of America diagnosed with GCA, where they reported a slight predilection for men of $66 \%$, similar to our findings $(62.5 \%)$. Both studies revealed that all cases of GCA were found at the posterior region of the mandible as the literature reports. They also described that GCA was predominant in caucasians $(60 \%)$ in contrast to our study that showed prevalence for non-caucasians (87.5\%). Regarding the average age, Hartman et al. found an average age of 40.7 compared to our finding of 46.8 [10].

Histophatologically, GCA showed significant tumor cell transformation in the cytoplasm caused by the presence of lysosomes, which corroborates with our results. These cells are usually stellate reticulum-like and present very coarse, granular and eosinophilic cytoplasm. The "granular change" is reported to happen due to a dysfunctional status of neoplastic cells, and the pathogenesis of this tumor seems to be age-related $[5,7,8]$. This granular change in classic ameloblastoma is a well-recognized phenomenon that was first seen by Krompecher in 1918, when the tumor cells were then called pseudo xanthomatous cells. However, whether the granular cell change in ameloblastoma is a degenerative process or a harbinger of more aggressive course remains unclear [11].

The differential diagnosis of GCA includes other oral lesions with a similar morphology of granular cell accumulation as granular cell myoblastoma, congenital epulis and granular cell ameloblastic fibromas. Although the morphology of all granular cells is similar, the tissue of origin is particular to each condition, and importantly these lesions present completely different biological pathways and should be discriminated from GCA $[3,5,11]$.

There has been considerable interest regarding the nature of granular cells in ameloblastoma ever since it was described. Studies have reported that these granular cells are derived from the odontogenic epithelium, rather than the connective tissue, as they highly express cytokeratins [12,13]. The transformation of neoplastic cells into granular cells has been currently attributed to "a degenerative process in a long standing lesion or a dysfunctional status of neoplastic cells" or a "metabolic change in response to aging phenomenon or an indicator of a more aggressive course" [2,9]. Ultra structural analysis have previously shown the presence of lysosomal aggregates in the cytoplasm of granular cells, and apoptotic cell fragments were detected between these cells within the neoplastic clusters, corroborating this hypothesis [13].

The basement membrane represents the barrier between the adjacent tissues, which provides mechanical support and plays important role in development and disease by regulating cell migration, adhesion and proliferation. Proteins of the basement membrane have been shown to be expressed in several types of odontogenic tumors that can define differences between their biological behavior [14]. An immune histochemical study of a single case of GCA has shown the moderate to strong protein expression of laminin and fibro nectin within the granular cells, whereas collagen IV was completely absent [15]. This corroborates similar findings in follicular ameloblastomas, and supports the hypothesis of age-related granular transformation, although further studies of GCA are required.

The biological behavior of GCA does not seem to differ from the other histologic subtypes of ameloblastoma, as it has been reported to be locally aggressive and recurrent. Reichart et al [8] have reported a $33.3 \%$ recurrence rate for this lesion, which was higher than the more common follicular, plexiform and acanthomatous subtypes. In Hartman's study [10], 11 of 15 patients (73\%) developed recurrent lesions of GCA. Unlike our case series study, no recurrence was detected within the 8 cases evaluated over more than three years of follow-up. This difference in recurrence rate is likely due to the surgical method performed, as the majority of cases received a less conservative treatment (surgical resection 87.5\%).

None of the GCA cases reported here was metastatic; however few cases of metastasis have been previously shown in the literature, spreading to cervical lymph nodes, lungs, thoracic or neck involvement. All cases presented long duration from 18 to 45 years [16-19], except from the latest case report of only two years progression [20], and most of them were recurrent and aggressive. It is still unknown what mechanism triggers metastasis in GCA, which is unusually metastatic. Therefore, performing early detection and diagnosis of this condition is essential to prevent long recurrences and potential malignancy.

This study reported eight retrospective cases of GCA with 
no history of recurrence or aggressive behavior. GCA is a rare condition with particular histopathological findings of granular cell transformation, whose clinical significance is still under investigation. The treatment and prognosis of GCA are similar to other subtypes of the ameloblastoma; however, case reports have been highlighting the potential risk of developing recurrences and metastasis if no early diagnosis and proper treatment are provided. A better understanding of the molecular pathogenesis of GCA would therefore improve diagnosis and therapeutic procedures.

\section{Acknowledgment}

This work was supported by the Post-Doctoral Program from CAPES - Brazilian Federal Agency for Support and Evaluation of Graduate Education within the Ministry of Education of Brazil.

\section{References}

1. Dave A, Arora M, Shetty VP, Saluja P. Granular cells in ameloblastoma: An enigma in diagnosis. Indian J Dent. 2015; 6: 211-4.

2. Wu YH, Chang JY, Wang YP, Chiang CP. Langerhans cells in granular cell ameloblastoma. J Formos Med Assoc. 2017; 116: 642-4.

3. Hunasgi S, Koneru A, Chauhan DS, Guruprasad Y. Rare giant granular cell ameloblastoma: a case report and an immunohistochemical study. Case Rep Dent. 2013; 2013: 372781.

4. Nikitakis NG, Tzerbos F, Triantafyllou K, Papadimas C, Sklavounou A. Granular cell ameloblastoma: an unusual histological subtype report and review of literature. J oral Maxillofac Res. 2010.

5. Motahhary P, Etebarian A, Asareh F. Granular cell type of a unicystic ameloblastoma: An unusual case and review of the literature. J Oral Maxillofac Pathol. 2014; 18: 331.

6. Jansari T, Samanta S, Trivedi P, Shah M. Granular cell ameloblastoma of mandible. Indian J Pathol Microbiol. 2014; 57: 305-307.

7. Yamunadevi A, Madhushankari GS, Selvamani M, Basandi PS, Yoithapprabhunath TR, Ganapathy N. Granularity in granular cell ameloblastoma. J Pharm Bioallied Sci. 2014; 6: 16-20.

8. Reichart PA, Philipsen HP, Sonner S. Ameloblastoma: biological profile of 3677 cases. Eur J Cancer B Oral Oncol. 1995; 31: 86-99.
9. Kameyama Y, Takehana S, Mizohata M, Nonobe K, Hara M, Kawai T, et al A clinicopathological study of ameloblastomas. Int J Oral Maxillofac Surg. 1987; 16: 706-712.

10. Hartman KS. Granular-cell ameloblastoma. Oral Surg Oral Med Oral Pathol. 1974; 38: 241-253.

11. Thillaikarasi R, Balaji J, Gupta B, Ilayarja V, Vani NV, Vidula B, et al. Cystic granular cell ameloblastoma. J Maxillofac Oral Surg. 2010; 9: 310-313.

12. Dina R, Marchetti C, Vallania G, Corinaldesi G, Eusebi V. Granular cell ameloblastoma. An immunocytochemical study. Pathol Res Pract. 1996: 192: 541-546.

13. Kumamoto $\mathrm{H}$, Ooya K. Immunohistochemical and ultrastructural investigation of apoptotic cell death in granular cell ameloblastoma. J Oral Pathol Med. 2001; 30: 245-250.

14. Henriques ÁC, Vasconcelos MG, Galvão HC, de Souza LB, de Almeida Freitas R. Comparative analysis of the immunohistochemical expression of collagen IV, MMP-9, and TIMP-2 in odontogenic cysts and tumors. Oral Surg Oral Med Oral Pathol Oral Radiol Endod. 2011; 112: 468-475.

15. Lapthanasupkul P, Poomsawat S, Chindasombatjaroen J. Investigation of basement membrane proteins in a case of granular cell ameloblastoma. Int J Oral Sci. 2012; 4: 45-9.

16. Cranin AN, Bennett J, Solomon M, Quarcoo S. Massive granular cell ameloblastoma with metastasis: report of a case. J Oral Maxillofac Surg. 1987; 45: 800-804

17. Hoke HF, Harrelson AB. Granular cell ameloblastoma with metastasis to the cervical vertebrae. Observations on the origin of the granular cells. Cancer. 1967; 20: 991-999.

18. Tsukada Y, Delapava S, Pickren JW. Granular-cell ameloblastoma with metastasis to the lungs: report of a case and review of the literature. Cancer. 1965: 916-25.

19. Takahashi K, Kitajima T, Lee M, Iwasaki N, Inoue S, Matsui N, et al. Granula cell ameloblastoma of the mandible with metastasis to the third thoracic vertebra. A case report. Clin Orthop Relat Res. 1985; 197: 171-180.

20. Bansal A, Bhatnagar A, Saxena S. Metastasizing granular cell ameloblastoma. J Oral Maxillofac Pathol. 2012; 16: 122-124. 